\title{
Adaptabilidade e estabilidade de genótipos de milho avaliadas por meio do modelo de regressão
}

\author{
Adaptability and stability of corn genotypes evaluated through \\ regression models
}

\section{Osvaldo Toshyiuki Hamawaki ${ }^{1}$ Patrícia Guimarães Santos ${ }^{2}$}

\section{RESUMO}

$O$ objetivo deste trabalho foi avaliar a estabilidade $e$ a adaptabilidade de cultivares de milho utilizando a metodologia de CRUZ et al. (1989). Foram avaliadas cultivares de ciclo precoce e normal, em dois ensaios conduzidos por três anos agrícolas em Capinopólis- $M G$, em quatro épocas de semeadura. $O$ delineamento experimental foi em blocos casualizados com três repetições, cada parcela foi constituída de quatro linhas de cinco metros, espaçadas de $90 \mathrm{~cm}$. Foram obtidos dados de produtividade de grãos e empregada a metodologia de Cruz para estimar os parâmetros de adaptabilidade e estabilidade das cultivares de milho. A cultivar ideal preconizada por Cruz, média alta, $\hat{b}_{1}<1, \hat{b}_{1}+\hat{b}_{2}>1 e$ variância dos desvios de regressão próximo ou igual a zero, não foi encontrada entre os materiais avaliados. Também não foi identificado nenhum material menos exigente nos ambientes desfavoravéis. De maneira geral, o material que mais se destacou foi o híbrido $G 551$, com média alta, boa resposta à melhoria do ambiente e de comportamento estável. A variedade BR 106 apresentou desempenho igual e até mesmo superior aos de alguns híbridos comerciais.

Palavras-chave: Zea mays, cultivares, interação genótipo $x$ ambientes.

\section{ABSTRACT}

The objective of this study was-to evaluate the adaptability and stability of corn cultivars used methodology of CRUZ et al. (1989). Early and normal cultivars were evaluated in two experiments conducted in three agricultural years a in Capinopólis-MG in four disting sowing times. The experimental design was a completely randomized block with three replications, the plots were constituted of four rows of five meters of length, spaced $0.9 \mathrm{~m}$. Yield grain data were obtained and utilized the methodology of Cruz for estimated the parameters of adaptability and stability of corn cultivars. The ideal cultivar extolled for Cruz, high mean, $\hat{b}_{1}<1, \hat{b}_{1}+\hat{b}_{2}>1$ and variance of the regression deviations near or equal to zero, were not found between tested materials. Also, material less exacting in unfavorable environment was not found. In general, the material that more detached was the hybrid $G 551$, with high mean, good responses to improved environment and stability. The variety BR 106 presented performance equal and even superior to some hybrids.

Key words: Zea mays, cultivars, genotype $x$ environment interaction

\section{INTRODUÇÃO}

O milho é produzido em quase todo o território brasileiro, nas mais diferentes regiões e com os mais diferentes sistemas de produção. Ante a essa diversidade de ambiente de cultivo, é esperada a ocorrência de uma forte interação genótipo x ambiente. Vários trabalhos utilizando estimativas de parâmetros de adaptabilidade e estabilidade têm sido realizados visando identificar cultivares mais adaptadas. Segundo MAGALHÃES \& PAIVA (1997) o uso de cultivares adaptadas é uma das poucas formas de conseguir acréscimo de produção sem custo adicional.

${ }^{1}$ Engenheiro Agrônomo, Doutor, Universidade Federal de Uberlândia (UFU), Instituto de Ciências Agrárias.

${ }^{2}$ Engenheiro Agrônomo, Doutor, (UFU), Instituto de Ciências Agrárias, CP 593, 38400-902, Uberlândia, MG. Email: pgsantos@umuarama.ufu.br. Autor para correspondência. 
A identificação de cultivares com maior estabilidade fenotípica tem sido uma alternativa muito utilizada para atenuar os efeitos da interação genótipos $\mathrm{x}$ ambientes e tornar o processo de recomendação de cultivares mais seguro. Existe uma série de metodologias para esse fim e constantemente surgem novas opções. Cada uma delas tem suas vantagens e desvantagens. Detalhes sobre elas podem ser encontrados em LIN et al. (1986).

A regressão é um processo muito utilizado no estudo da adaptabilidade e estabilidade de cultivares. Ela foi inicialmente proposta por alguns pesquisadores, porém recebeu mais notoriedade através dos trabalhos de FINLAY \& WILKINSON (1963) e EBERHART \& RUSSELL (1966).

Outra metodologia utilizando regressão foi proposta por VERMA et al. (1978). A metodologia prevê a estimativa de duas retas de regressão, uma abrangendo os ambientes desfavoráveis e a outra os ambientes favoráveis. Essa metodologia foi aperfeiçoada por SILVA \& BARRETO (1985) e posteriormente por CRUZ et al. (1989). Ela baseia-se em uma análise de regressão bissegmentada e tem como parâmetros de estabilidade e adaptabilidade a média $\left(\mathrm{b}_{0 \mathrm{i}}\right)$, a resposta linear aos ambientes desfavoráveis $\left(b_{1 i}\right)$, a resposta linear aos ambientes favoráveis $\left(b_{1 i}+\right.$ $\left.b_{2 i}\right)$ e o desvio da regressão $\left(\sigma^{2}\right.$ di $)$. Utiliza-se um modelo de regressão em que $\mathrm{I}_{\mathrm{j}}$ e $\mathrm{T}\left(\mathrm{I}_{\mathrm{j}}\right)$ fazem o papel de variável independente. Conforme comentam VENCOVSKY \& BARRIGA (1992), por esse modelo pode-se detectar, por exemplo, se um tratamento tem comportamento diferente nos ambientes desfavoráveis e favoráveis. Assim, se $b_{1 i}$ for baixo e $b_{1 i}+b_{2 i}$ for alto, tem-se a indicação de que o genótipo não é afetado pela deterioração da qualidade ambiental, mas é responsivo se as condições ambientais melhoram. Tal detalhamento não é possível pelo método de EBERHART \& RUSSELL(1966).

Segundo CRUZ \& CASTOLDI (1991) a adaptabilidade refere-se à capacidade dos genótipos aproveitarem vantajosamente o estímulo do ambiente. Já a estabilidade refere-se à capacidade dos genótipos mostrarem um comportamento altamente previsível em função do estímulo do ambiente. Para isso, são obtidas as estimativas de $\sigma^{2}$ di e $R^{2}$.

O método de Eberhart \& Russell tem sido empregado quando se dispõe de um número de ambientes acima de três. Com ambientes em número igual ou maior do que oito, pode-se adotar um modelo bissegmentado. De maneira geral, a análise da estabilidade, além das propriedades intrínsecas que possui, é uma maneira alternativa de estudar o fenômeno da interação genótipos $\mathrm{x}$ ambientes (VENCOVSKY \& BARRIGA, 1992).

Várias metodologias têm sido empregadas para avaliação da adaptabilidade e estabilidade de cultivares de milho. GONÇALVES et al. (1999) utilizaram as metodologias de LIN \& BINS (1988) e ANNICCHIARICO (1992) para avaliar o desempenho de variedades e híbridos de milho nos Estados de Goiás, Paraná e São Paulo, no período da safrinha. Ambas metodologias forneceram praticamente os mesmos resultados, identificando os híbridos Zeneca 8452, C 805 e Agromem 2012 como os mais estáveis.

A metodologia de CRUZ et al. (1989) tem sido utilizada por vários pesquisadores. GAMA et al. (2000) empregaram esta metodologia para estudar a adaptação de diferentes populações e cultivares de milho em diferentes regiões do País. Eles concluíram que os híbridos possuem maior adaptação (produtividade acima da média) que as populações e variedades; o C 901 foi o único híbrido que apresentou adaptabilidade ampla, apresentado o mesmo nível de resposta, tanto em ambientes favoráveis como desfavoráveis. Verificaram também que o híbrido simples (C 901) apresentou maior estabilidade que os híbridos triplos e duplos avaliados neste trabalho.

A adaptabilidade e estabilidade de cultivares e populações de milho no Nordeste têm sido estudadas por CARVALHO et al. (1999) e CARVALHO et al. (2000), utilizando a metodologia de CRUZ et al. (1989). No primeiro trabalho, foram identificados apenas três híbridos entre 14, com alta estabilidade e a maioria das variedades apresentou estável, destacando as variedades BR 106 e BR 5011. No segundo trabalho citado, os autores concluíram que as variedades BR 106, BR 5011 e BR 5004 podem aumentar a produtividade média dos sistemas de produção dos pequenos e médios produtores rurais. Quanto aos híbridos, destacaram-se o BR 3123, Agromem 2003, e Germinal 600 , pois apresentaram respostas positivas à melhoria ambiental, sendo, portanto, uma boa opção para os produtores do Nordeste que tenham condições de investir em tecnologia visando à melhoria do ambiente.

Diante do exposto, o presente trabalho teve como objetivo avaliar a estabilidade e adaptabilidade de cultivares de milho na região do Triângulo Mineiro utilizando a metodologia proposta por CRUZ et al. (1989).

\section{MATERIALEMÉTODOS}

O trabalho foi realizado no CEPET (Centro de Pesquisa do Triângulo), localizado no município de Capinópolis-MG. Foram realizados dois ensaios, um 
com 10 cultivares de ciclo precoce e o outro com 9 cultivares de ciclo normal. Os experimentos foram conduzidos nos anos agrícolas de 1990/91, 1991/92 e 1992/93 em quatro épocas de semeadura, que variaram de outubro a dezembro. A precipitação pluvial ocorrida no período de condução dos experimentos é mostrada na tabela 1.

O delineamanto experimental foi em blocos casualizados com três repetições. Cada parcela foi constituída de 4 linhas de 5 metros, espaçadas de 90 $\mathrm{cm}$. A adubação de plantio foi realizada utilizando 400 $\mathrm{kg} \mathrm{ha}^{-1}$ da formulação 08-28-16 + BR12 e a adubação de cobertura foi feita aos 35 dias após a semeadura quando se aplicou $400 \mathrm{~kg} \mathrm{ha}^{-1}$ da formulação 30-0-20. Os demais tratos culturais como controle de pragas e plantas daninhas foram realizados segundo as recomendações para a cultura do milho.

O caráter avaliado foi produtividade de grãos ( $\left.\mathrm{t} \mathrm{ha}^{-1}\right)$, obtida através da pesagem dos grãos de cada parcela, sendo feita a correção de umidade para $13 \%$.

A metodologia empregada para estimar os parâmetros de estabilidade e adaptabilidade foi a de CRUZ et. al. (1989), que é uma extensão da metodologia proposta por SILVA \& BARRETO (1985), tornando-a mais simples e com propriedades estatísticas mais adequadas aos propósitos do melhoramento. A metodologia baseia-se na análise de regressão bissegmentada e tem, como parâmetros de adaptabilidade, a média $\left(b_{0 i}\right)$ e a resposta linear aos ambientes desfavoráveis $\left(b_{1 i}\right)$ e aos ambientes favoráveis $\left(b_{1 i}+b_{2 i}\right)$. A estabilidade dos materiais é avaliada pelos desvios da regressão $\sigma^{2}$ de cada material, em função das ( kariações ambientăis.

O modelo adotado foi o seguinte:
$\mathrm{Y}_{\mathrm{ij}}=\mathrm{b}_{0 \mathrm{i}}+\mathrm{b}_{1 \mathrm{i}} \mathrm{I}_{\mathrm{j}}+\mathrm{b}_{2 \mathrm{i}} \mathrm{T}\left(\mathrm{I}_{\mathrm{j}}\right)+\delta_{\mathrm{ij}}+\boldsymbol{\varepsilon}_{i j}$ em que: $Y_{i j}$ : média da cultiva i no ambiente $\mathrm{j}$; $\mathrm{Ij}_{\mathrm{j}}$ : índice ambiental utilizado por EBERHART \&

Tabela 1 - Precipitação pluvial (mm) ocorrida durante os anos agrícolas de 1991/92 e 1992/93 em Capinopólis-MG

\begin{tabular}{|c|c|c|}
\hline \multirow{2}{*}{ MESES } & \multicolumn{2}{|c|}{ ANO } \\
\hline & $1991 / 92$ & $1992 / 93$ \\
\hline OUTUBRO & 75,1 & 182,9 \\
\hline NOVEMBRO & 52,4 & 187,3 \\
\hline DEZEMBRO & 148,7 & 356,3 \\
\hline JANEIRO & 300,6 & 59,8 \\
\hline FEVEREIRO & 228,5 & 362,8 \\
\hline MARÇO & 179,2 & 72,3 \\
\hline ABRIL & 93,6 & 86,5 \\
\hline MAIO & 58,6 & 27,9 \\
\hline JUNHO & 0 & 27,4 \\
\hline TOTAL & 1136,7 & 1363,2 \\
\hline
\end{tabular}

RUSSELL (1966). Tem-se T( $\left.\mathrm{I}_{j}\right)=0$ se Ij é menor que zero e $\mathrm{T}(\mathrm{I} j)=\mathrm{Ij}_{\mathrm{j}}-\mathrm{I}+\mathrm{se} \mathrm{Ij}_{\mathrm{j}}>0$ sendo $\mathrm{I}+\mathrm{a}$ média dos índices $\mathrm{Ij}$ positivos; $b_{0 \mathrm{i}}$ : média geral da cultivar $\mathrm{i} ; \mathrm{b}_{1 \mathrm{i}}$ :coeficiente de regressão linear associado a ambientes desfavoráveis; $\mathrm{b}_{1 \mathrm{i}}+\mathrm{b}_{2 \mathrm{i}}$ : coeficiente de regressão linear associado a ambientes favoráveis; $\delta_{\mathrm{ij}}$ : desvio de regressão linear; $\bar{\varepsilon}_{i j}$ : erro médio associado à média.

\section{RESULTADOS E DISCUSSÃO}

O resumo da análise de variância conjunta dos experimentos de avaliação das cultivares de milho encontra-se na tabela 2, ciclo precoce e normal. Observase que não houve interação significativa entre as cultivares de ciclo precoce e ambientes, evidenciando que os materiais comportaram-se de forma semelhante nos ambientes estudados. A interação significativa entre materiais de ciclo normal e os ambientes pode ser constatada neste trabalho. Isso mostra que é preciso minimizar os efeitos desta interação e ter maior previsibilidade de comportamento dos materiais. É necessário também identificar os materiais mais estáveis e/ou adaptados às condições específicas de ambiente.

Neste trabalho, utilizou-se a metodologia proposta por CRUZ et al. (1989) para estimar os parâmetros de adaptabilidade e estabilidade dos materiais de ciclo normal. Nesta metodologia, procuramse materiais produtivos, estavéis em ambientes desfavoráveis e muito responsivos à melhoria do ambiente.

A produtividade média das cultivares variou de 4,00 e 4,12 t ha ${ }^{-1}$ para os híbridos AG 162 e AGM

Tabela 2 - Resumo da análise de variância conjunta da produtividade de grãos $\left(\mathrm{t} \mathrm{ha}{ }^{-1}\right)$ de milho de 10 cultivares de ciclo precoce e 9 de ciclo normal avaliadas em três anos e quatro épocas.

\begin{tabular}{lcc}
\hline Precoce & & \\
\hline Fontes de variação & GL & QM \\
Ambientes (A) & 11 & $44,06^{* *}$ \\
Cultivares ( C ) & 9 & $12,17^{* *}$ \\
A x C & 99 & $0,76^{\text {ns }}$ \\
Resíduo & 238 & 1,07 \\
\hline Média geral & 4,69 & \\
CV (\%) & 22 & \\
\hline Ciclo normal & & $66,16^{* *}$ \\
\hline Ambientes (A) & 10 & $7,42^{* *}$ \\
Cultivares ( C ) & 8 & $0,93^{* *}$ \\
A x C & 80 & 0,55 \\
Resíduo & 196 & \\
\hline Média geral & 4,72 & \\
CV (\%) & 16 & \\
\hline
\end{tabular}


1022 a 5,35 e 5,31 tha-1 para os híbridos G 551 e G 500, respectivamente. Os demais materiais apresentaram comportamento intermediário, e diferenças significativas entre si (Tabela 3).

As estimativas do parâmetro $\hat{b}_{1}$, que corresponde à resposta linear do genótipo às variações nos ambientes desfavoráveis, variaram de 0,83 a 1,30, apenas os híbridos triplos P 3210 e P 3232 apresentaram-se como exigentes em ambientes desfavoravéis $\left(\hat{b}_{1}>1\right)$. Os demais materiais apresentaram $b=1$, evidenciando adaptabilidade ampla; não foi possível identificar nenhum material com adaptação a ambiente desfavorável. Com relação à resposta nos ambientes favoráveis, somente o híbrido G 551 apresentou resposta à melhoria do ambiente ao $\left(\hat{b}_{1}+\hat{b}_{2}>1\right)$, contrário do híbrido AG 162 com valor menor que 1 . Os materiais com $\hat{b}_{1}+\hat{b}_{2}=1$ respondem proporcionalmente às mudanças de ambientes. Quanto à estabilidade dos genótipos, apenas os híbridos AGM 1022 e G 500, não apresentaram comportamento previsível. É importante salientar que o grau de previsibilidade não deve comprometer a indicação destes híbridos, uma vez que a estimativa de $\mathrm{R}^{2}$ esteve acima de $80 \%$, para todas cultivares. Este parâmetro indica que houve um bom ajustamento dos dados à reta de regressão (CRUZ et al., 1989).

A cultivar ideal preconizada por Cruz, média alta, $\hat{b}_{1}<1, \hat{b}_{1}+\hat{b}_{2}>1$ e variância dos desvios de regressão próximo ou igual a zero, não foi encontrada entre os materiais avaliados. Também não foi identificado nenhum material menos exigente nos ambientes desfavoravéis (Figura 1). De maneira geral, o material que mais destacou foi o híbrido G 551, com média alta, boa resposta à melhoria do ambiente e de comportamento estável. A variedade BR 106 apresentou desempenho igual e até mesmo superior aos de alguns

Tabela 3 - Estimativas das médias ${ }^{1}$ e dos parâmetros de adaptabilidade e estabilidade cultivares de milho de ciclo normal avaliadas em três locais e quatro épocas, obtidos pelo método de Cruz et al. (1989).

\begin{tabular}{|c|c|c|c|c|c|c|c|c|}
\hline \multirow[t]{2}{*}{ Cultivares } & \multicolumn{3}{|c|}{ Média } & \multirow[t]{2}{*}{$\mathrm{B}_{1}$} & \multirow[t]{2}{*}{$\mathrm{b}_{2}$} & \multirow[t]{2}{*}{$b_{1}+b_{2}$} & \multirow[t]{2}{*}{ QM desvio } & \multirow[t]{2}{*}{$\mathrm{R}^{2}(\%)$} \\
\hline & Geral & Desfav. & Fav. & & & & & \\
\hline C 125 & $4,73 \mathrm{c}$ & 3,16 & 5,63 & $0,96^{\mathrm{ns}}$ & $-0,37^{\mathrm{ns}}$ & $0,58^{\mathrm{ns}}$ & $0,58^{\mathrm{ns}}$ & 93,2 \\
\hline C 135 & $5,03 \mathrm{~b}$ & 3,63 & 5,83 & $0,83^{\text {ns }}$ & $0,30^{\mathrm{ns}}$ & $1,13^{\mathrm{ns}}$ & $0,17^{\mathrm{ns}}$ & 97,5 \\
\hline AGM 1022 & $4,12 \mathrm{e}$ & 2,67 & 4,95 & $0,91^{\mathrm{ns}}$ & $-0,20^{\mathrm{ns}}$ & $0,72^{\mathrm{ns}}$ & $1,10^{*}$ & 87,0 \\
\hline AG 162 & $4,00 \mathrm{e}$ & 2,40 & 4,88 & $0,98^{\text {ns }}$ & $-0,66^{*}$ & $0,33^{*}$ & $0,76^{\mathrm{ns}}$ & 91,5 \\
\hline G551 & $5,35 \mathrm{a}$ & 3,86 & 6,21 & $0,88^{\text {ns }}$ & $1,42 * *$ & $2,30 * *$ & $0,98^{\mathrm{ns}}$ & 91,4 \\
\hline G 500 & $5,31 \mathrm{a}$ & 3,82 & 6,17 & $1,04^{\mathrm{ns}}$ & $0,36^{\mathrm{ns}}$ & $1,40^{\mathrm{ns}}$ & $1,07 *$ & 90,8 \\
\hline
\end{tabular}

${ }^{1}$ Médias não seguidas pela mesma letra diferem entre si pelo teste de Tukey a $1 \%$ de probabilidade de erro e agrupadas pelo método de Fasoulas (1983).

$* \mathrm{e} * *$ Significativamente diferente de um, para $\mathrm{b}_{1}$ e $\mathrm{b}_{1}+\mathrm{b}_{2}$ e de zero para $\mathrm{b}_{2}$, a $5 \%$ e $1 \%$ de probabilidade de erro, respectivamente, pelo teste de t e pelo teste F em Qmdesvio. 
híbridos comerciais (AGM 1022 e AG 162). Esta variedade tem bom potencial produtivo e é muito utilizada em programas de melhoramento de milho.

\section{CONCLUSÕES}

O comportamento das cultivares de ciclo normal foi afetado pelos diferentes ambientes testados.

O híbrido que mais se destacou foi o G 551, com média alta, adaptação a ambientes favoravéis e boa estabilidade.

\section{REFERÊNCIASBIBLIOGRÁFICAS}

ANNICCHIARICO, P. Cultivar adaptation and recommendation from alfafa trials in Northern Italy. Journal Genetic and Breeding, Lodi, v.46, n.3, p.269-278, sep, 1992.

CARVALHO,H.W.L. et al. Adaptabilidade e estabilidade de produção de cultivares de milho no Nordeste Brasileiro. Pesquisa Agropecuária Brasileira, Brasília, v.34, n.9, p.1581-1591, set, 1999.

CARVALHO, H.W.L. et al. Adaptabilidade e estabilidade de cultivares de milho no Nordeste Brasileiro. Pesquisa Agropecuária Brasileira, Brasília,v.35, n.6, p.1115-1123, jun, 2000

CRUZ, C.D.; CASTOLDI, F.L. Decomposição da interação genótipos $\mathrm{x}$ ambientes em partes simples e complexa. Revista Ceres, Viçosa, v.38, n.219, p.422-430, 1991.

CRUZ, C.D.; TORRES, R.A. de A.; VENCOVSKY, R. An alternative approach to the stability analysis proposed by Silva and Barreto. Revista Brasileira de Genética, Ribeirão Preto, v. 12 , n.3, p.567-80, 1989.

EBERHART, S.A.; RUSSELL, W.A. Stability parameters for comparing varieties. Crop Sci, Madison, v.6, n.1, p.36-40, Jan/Feb, 1966.
FASOULAS, A.C. Rating cultivars and trials in applied palnt breeding. Euphytica, Wageningen, v.32, n.3, p. 939-943, nov, 1983.

FINLAY, K.W.; WILKINSON, G.N. The analysis of adaptation in a plant breeding programme. Australian Journal of Agricultural Research, Melbourne, v.14, p.742-754, 1963.

GAMA, E.E.G. et al. Estabilidade da produção de germoplasma de milho avaliados em diferentes regiões do Brasil. Pesquisa Agropecuária Brasileira, Brasília. v.35, n.6, p. 1143-1149, jun, 2000 .

GONÇALVES, F.M.A. et al. Importância das interações cultivares $\mathrm{x}$ locais e cultivares $\mathrm{x}$ anos na avaliação de milho na safrinha. Pesquisa Agropecuária Brasileira, Brasília, v.34, n.7, p.1175-1181, jul, 1999 .

LIN, C.S.; BINNS,M.R. A method of analysing cultivars x location $x$ year experiments: new stability parameter. Theoretical Applied Genetics, Berlim, v.76, p.425-430, 1988

LIN, C.S.; BINNS, M.R.; LEFKOVITCH, L.P. Stability analysis where do we stand? Crop Science, Madison, v.26, p.894-900, 1986.

MAGALHÃES, P.C.; PAIVA, E. Fisiologia da produção. In: CRUZ, J.C. et al. Recomendações técnicas para o cultivo do milho. Brasília : EMBRAPA, 1997. p.85-96.

SILVA, J.G.C.; BARRETO, J.N. Aplicação de regressão linerar segmentada em estudo da interação genótipo $\mathrm{x}$ ambiente. In: SIMPÓSIO DE ESTATÍSTICA APLICADA À EXPERIMENTAÇÃO AGRONÔMICA, 1., 1985 , Piracicaba. Resumos... Campinas : Cargill, 1985. p.49. 50

VENCOVSKY, R.; BARRIGA, P. Genética biométrica aplicada no fitomelhoramento. Ribeirão Preto : Sociedade Brasileira de Genética, 1992. 496p.

VERMA, M.M.; CHAHAL, G.S.; MURTY, B.R. Limitations of conventional regression analysis: a proposed modification. Theoretical and Applied Genetics, Berlim, v.53, p.89-91, 1978. 